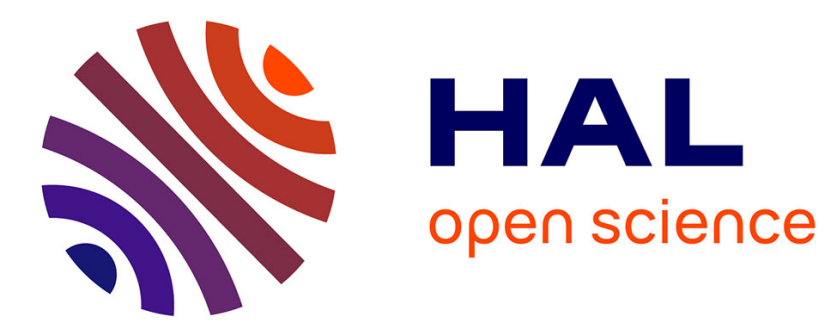

\title{
New dielectric properties of glasses at very low temperatures
}

\author{
G. Frossati, R. Maynard, R. Rammal, D. Thoulouze
}

\section{To cite this version:}

G. Frossati, R. Maynard, R. Rammal, D. Thoulouze. New dielectric properties of glasses at very low temperatures. Journal de Physique Lettres, 1977, 38 (6), pp.153-156. 10.1051/jphyslet:01977003806015300 . jpa-00231348

\section{HAL Id: jpa-00231348 https://hal.science/jpa-00231348}

Submitted on 1 Jan 1977

HAL is a multi-disciplinary open access archive for the deposit and dissemination of scientific research documents, whether they are published or not. The documents may come from teaching and research institutions in France or abroad, or from public or private research centers.
L'archive ouverte pluridisciplinaire HAL, est destinée au dépôt et à la diffusion de documents scientifiques de niveau recherche, publiés ou non, émanant des établissements d'enseignement et de recherche français ou étrangers, des laboratoires publics ou privés. 
Classification

Physics Abstracts

8.720

\title{
NEW DIELECTRIC PROPERTIES OF GLASSES AT VERY LOW TEMPERATURES
}

\author{
G. FROSSATI, R. MAYNARD, R. RAMMAL and D. THOULOUZE \\ Centre de Recherches sur Les Très Basses Températures, C.N.R.S., \\ 166 X, 38042 Grenoble Cedex, France
}

(Reçu le 17 janvier 1977, accepté le 18 février 1977)

\begin{abstract}
Résumé. - La constante diélectrique de borosilicates vitreux a été mesurée à très basse fréquence, jusqu'à $5 \mathrm{mK}$. Une variation en Log $T / \omega$ et une réponse non linéaire en champ ont été trouvées et expliquées par une distribution très large des temps de relaxation de réorientation des dipôles. Les fréquences caractéristiques de l'effet tunnel sont bien inférieures à celles déduites des propriétés acoustiques.
\end{abstract}

\begin{abstract}
The very low frequency dielectric constant of borosilicate glasses has been measured down to $5 \mathrm{mK}$. A new law in $\log T / \omega$ and a non-linear field effect have been found and are explained by a very broad distribution of the relaxation times for the reorientation of the dipoles. The characteristic tunneling frequencies are lower than the corresponding ones deduced from the acoustic properties.
\end{abstract}

In amorphous substances, the existence of low energy excitations is now well established from their thermal [1] and dynamic [2], [3] properties at very low temperatures. Even though the nature of these excitations is still unknown, they are phenomenologically well described as two-level systems [4] strongly coupled to the phonon via elastic dipoles. The high frequency dielectric properties above $0.3 \mathrm{~K}$ have revealed a strong dependence on the concentration of dipolar impurities imbedded in glasses $\left(\mathrm{OH}^{-}\right.$ions in vitreous $\left.\mathrm{SiO}_{2}\right)$. This nonintrinsic dielectric behaviour contrasts with the elastic properties which are not sensitive to the presence of impurities. The comparison between the elastic and dielectric properties motivated the present study of the very low temperature dielectric constant of glasses. Moreover, except for one measurement on a glass ceramic capacitance thermometer [5], no systematic study has been performed for very low frequencies and temperatures.

We have measured the very low frequency dielectric constant of a borosilicate glass at temperatures varying from $5 \mathrm{mK}$ to $1 \mathrm{~K}$, for different excitation levels and frequencies. The borosilicate BK 7 glass was chosen since its dielectric properties had been previously studied at higher frequencies and temperatures [3]. The sample, in the form of a disc $0.3 \mathrm{~mm}$ thick and about $1 \mathrm{~cm}^{2}$, was metallized on both sides in the form of a central electrode surrounded by a guard ring. Its capacitance was measured with a bridge using a ratio transformer, a reference quartz capacitor in the ${ }^{4} \mathrm{He}$ bath and a synchronous detector. Excitation levels between $0.9 \mathrm{mV}$ and $0.910 \mathrm{~V}$ were used at frequencies of $130 \mathrm{~Hz}, 1.3$ and $13 \mathrm{kHz}$. The capacitance to be measured was immersed in the mixing chamber of a dilution refrigerator working continuously down to $6 \mathrm{mK}$. The temperature was given by the a.c. susceptibility of a powdered CMN cylinder and by calibrated silicon thermometers. The power dissipated in the sample varied between $10^{-18} \mathrm{~W}$ and $10^{-12} \mathrm{~W}$ according to the voltage applied. Knowing the magnitude of the diffusivity and the Kapitza coefficients of the glasses, a maximum value of $0.2 \mathrm{mK}$ can be estimated for the overheating at $10 \mathrm{mK}$.

The results are shown in figure 1 for the intermediate frequency of $1300 \mathrm{~Hz}$. The behaviour of the dielectric constant $\varepsilon$ is the same for the other frequencies. For the smallest electrical fields, we may note a logarithmic variation of $\varepsilon$ over the whole temperature range, $\varepsilon \sim \ln T / \omega$, as may be seen on figure 2 . On the low temperature side, $\varepsilon$ depends strongly on the magnitude of the electrical field $F$, and increases at the lowest temperatures. Qualitatively similar field dependences have also been observed in Kapton and polyethylene using metallized electrodes in the 


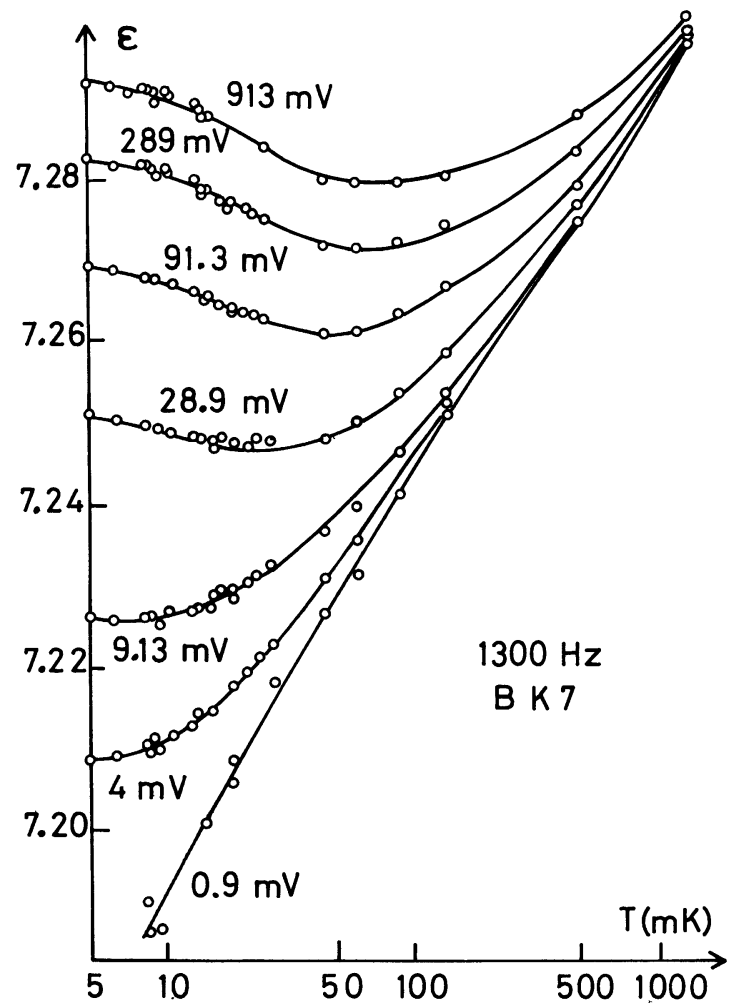

FIG. 1. - The measured dielectric constant $\varepsilon$ of BK 7 glass as a function of temperature at $1300 \mathrm{~Hz}$ for different voltages.

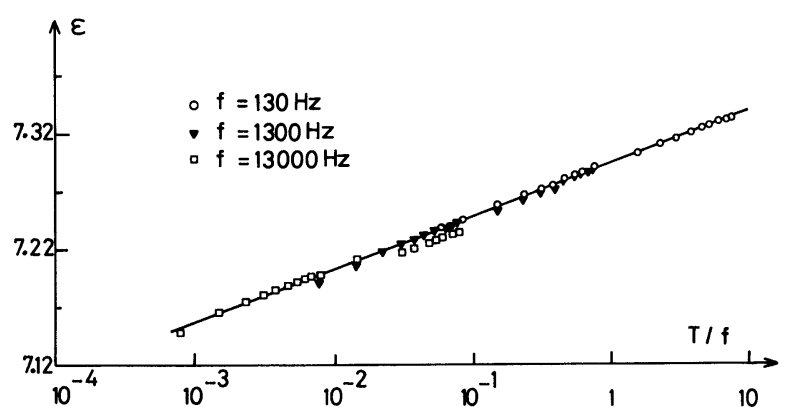

FIG. 2. - The universal behaviour of the dielectric constant $\varepsilon$ as a logarithmic function of the reduced variable $T / f$, with $T$ in $\mathrm{mK}$ and $\omega=2 \mathrm{Mf}$.

first case, and indium ones in the second case $\left({ }^{1}\right)$. With the same electrodes, no field dependence has been detected at $4.2 \mathrm{~K}$, even for excitation levels up to $20 \mathrm{~V}$.

These features can be understood in the framework of a non-equilibrium theory, assuming that the contribution of the elementary dipoles is reduced when their relaxation time becomes longer than the time of the experiments at very low temperature.

( ${ }^{1}$ Le Gilchrist, J. G., Private Communication.
For the weak field regime, the quantitative analysis starts from the standard relaxation formula :

$$
\alpha=\left\langle\frac{\alpha_{0}}{1+\omega^{2} \tau^{2}}\right\rangle
$$

where $\alpha_{0}$ is the real part of the polarizability of the dipole $\mu, \omega$ the frequency of a.c. electrical field, $\tau$ the relaxation time of the dipole and $\alpha$ the macroscopic polarizability. The mechanism of relaxation and the choice of the dipole random variables are the basic ingredients of the present model. We suppose here that the reorientation of the dipoles by the electric field is due to the tunnelling motions of ions through a potential barrier $V$ [4]. In addition, due to the disorder of the structure, the most probable situation involves an asymmetric potential or a zero-field splitting $\Delta$. At very low temperature, the dominant mechanism of relaxation is the so-called one phonon induced tunnelling leading to the following expression for the relaxation time :

$$
\tau=\frac{a}{\Delta} \mathrm{e}^{2 \lambda} \tanh \left(\frac{\Delta}{2 T}\right)
$$

where $\lambda$ is the WKB tunnelling parameter $\lambda=(V / W)^{1 / 2}$ and $W=\left(h^{2} / 2 m\right) \times d^{-2}, d$ the depth of the barrier and $m$ the mass of the ion.

This formula is not the standard one $\left({ }^{1}\right)$ used in the context of the ultrasonic propagation of glasses. It involves the unrenormalized asymmetry energy $\Delta$ instead of the exact energy $\left(\Delta^{2}+C^{2} \mathrm{e}^{-2 \lambda}\right)^{1 / 2}$ where $C$ is the curvature of the potential minima. Here we assume that the tunnelling coupling energy $C \mathrm{e}^{-\lambda}$ is almost always negligible compared to $\Delta$ or $k T$, except indeed in the inverse relaxation time where the contribution is calculated to the lowest order in $\mathrm{e}^{-2 \lambda}$ (typically $C \leqslant 10 \mathrm{mK}$ ). This hypothesis makes reference to the model of strong elastic deformation around the defect or the polaron model of dipolar reorientation [6].

The polarizability of permanent dipoles under the influence of the zero field splitting $\Delta$ is given by :

$$
\alpha_{0}^{\prime}=\frac{1}{3} \frac{\mu^{2}}{T \cosh ^{2}\left(\frac{\Delta}{2 T}\right)} .
$$

Assuming that in glasses the variables $\Delta$ and $\lambda$ are randomly distributed between 0 and $\infty$ (ergodic variables), the macroscopic polarizability is then obtained from [1], [2] and [3] :

$\alpha^{\prime}=\frac{1}{3} \frac{\mu^{2}}{T} n \int_{0}^{\infty} \mathrm{d} \Delta \mathrm{d} \lambda \frac{1}{\cosh ^{2}\left(\frac{\Delta}{2 T}\right)} \frac{1}{1+\dot{\omega}^{2} \tau^{2}}$

where $n$ is the constant density of dipoles in the $\Delta$, $\lambda$ plane. 
The double integration leads to the expressions :

$$
\alpha^{\prime}= \begin{cases}\frac{\pi^{2}}{18} n \mu^{2}\left(\frac{T}{T_{\omega}}\right)^{2} & T \ll T_{\omega}=\frac{a \omega}{2} \\ \frac{2}{3} n \mu^{2}\left(0.18+\ln \frac{T}{T_{\omega}}\right), & T \gg T_{\omega}=\frac{a \omega}{2} .\end{cases}
$$

It should be emphasized that this result originates in the assumption of $T W O$ random variables, only one of which $\Delta$ is scaled by the temperature. The choice of one single ergodic variable (rigid double well potential) where both the tunnelling coupling energy $\varepsilon$ and the asymmetry $\Delta$ could vary on the range of $k T$ would have led to $\ln \left(T / \omega^{1 / 3}\right)$, a law which is not observed here. Then the assumption of weak tunnelling makes the distribution of the relaxation times of the dipoles markedly different from that of two-level systems (the same hypothesis for the acoustic properties would lead to the wrong temperature dependence for thermal conductivities or acoustic absorption). The $\ln (T / \omega)$ law is the quantum tunnelling counterpart of the classical activation process which gives $T \ln \left(\omega^{-1}\right)$ in the theory of magnetic relaxation of grains [7] or Bloch walls.

The linearized version of the Clausius-Mossotti law can be used to establish the relation between the measured dielectric constant $\varepsilon$, the host constant $\varepsilon_{0}$ $\left(\varepsilon_{0} \simeq 7\right)$ and the polarizability $\alpha^{\prime}$ :

$$
\varepsilon=\varepsilon_{0}+(4 \pi / 9)\left(\varepsilon_{0}+2\right)^{2} \alpha^{\prime} .
$$

From the slope in figure 2 one finds

$$
n \mu^{2}=2.7 \times 10^{-4} \text {. }
$$

For $\mu=1$ Debye $\left(=10^{-18} \mathrm{erg}\right)$ one derives

$$
n=2.7 \times 10^{32} \mathrm{~cm}^{-3} \mathrm{ergs}^{-1} \text {. }
$$

This value which is not far from the density of two-level systems shows that the dipoles can contribute to the specific heat by the same amount as the two-level defects.

The contribution of the induced dipoles via the resonant interaction of the electromagnetic field at high frequency [3] seems to be at least one order of magnitude smaller than the contribution of the permanent dipoles. Moreover the frequency dependence of the measured dielectric constant at low frequency and low temperature confirms the weakness of the induced dipoles cortribution.

The threshold of the non-linear behaviour starts at very low intensities of the electrical field, such that $\mu F / k T \simeq 10^{-3}$ for $\mu=1$ Debye. This situation is very unusual and cannot be explained by antiferroelectric interaction among the dipoles : even if the interaction energy $J$ was a random variable, a field dependence would be expected when $\mu F \gtrsim J$ and $J \gtrsim k T$. Actually so low a threshold reminds us of the first magnetization curve in the Rayleigh domain and can be understood, at least qualitatively, in this way.

Basically such non-linearity resides in the field dependence of the relaxation time $\tau$, which prevents the use of formula (1) which is valid only in the framework of the linear response theory. A simple estimation of the non-linear contribution can be obtained by describing the response of the dipoles in terms of rectangular hysteresis loops characterized by the critical fields $F a$ and $F b$. In the $\omega \rightarrow \infty$ limit, only the systems for which $\tau \rightarrow 0$ will be observable; the critical fields $F a$ and $F b$ are nothing else than the barrier heights measured from the minima of the double well potential : $F a=V$ and $F b=\Delta+V$. For this condition, only the dipoles for which the critical fields are less than the values $\xi F$ (where $\xi$ is a numerical factor) can contribute to the polarization, such that $|\Delta+V| \lesssim \xi \mu F$ and $V \lesssim \xi \mu F$. With the previous assumptions about the ergodic variables $n(\Delta, \lambda)=n$, the density of dipoles in the $\Delta, V$ plane is

$$
n(\Delta, V)=n / 2 \sqrt{V W}
$$

and the number of the contributing dipoles is $2 \xi^{3 / 2} \mu F n(\mu F / W)^{1 / 2}$. The polarizability

$$
\text { - } \quad \alpha^{\prime}=2 \xi^{3 / 2} \mu^{2} n(\mu F / W)^{1 / 2}
$$

exhibits an unusual non-linear variation in $F^{1 / 2}$ which is verified experimentally at very low field. From the slope of $\varepsilon v s$. $F^{1 / 2}$, and taking

$$
n \mu^{2}=2.7 \times 10^{-4} \text {, }
$$

we find for $\xi=10, W=1.8 \times 10^{-2} \mathrm{~K}$, a value which corresponds to the tunnelling of about 10 molecules through $1 \AA$. This result must not be considered too strictly since $\xi$ has been chosen in a rather arbitrary way to take into account the finite value of the experimental frequency, but nevertheless it indicates that the mass of the tunnelling group of atoms is large, thus leading to small tunnelling effects. This approach is however only valid in the high frequency regime at very low temperature. A complete treatment of such a non-linear relaxation equation at any frequency and temperature, which requires the detailed expression of the relaxation time as a function of $F$, will be published shortly [9].

In conclusion, the new law $\ln T / \omega$ found for the real part of the dielectric constant reflects a broad distribution of relaxation times of the electrical dipoles around the low frequencies of the measurement. This distribution involves weak tunnelling frequencies and differs from the standard assumption of the strong tunnelling frequencies which is usually invoked for explaining the acoustical properties of glasses. These low values of the tunnelling frequencies probably imply a stronger interaction of the dipoles 
with the lattice than for the usual two-level systems. The role of these relaxations is still more efficient in the strong non-linearities of the dielectric constant. The description of this new behaviour in terms of hysteresis loops implies remanent polarizations, the measurement of which is in progress.

We are grateful to Dr. R. Tournier for suggesting the analogy with the non-linear behaviour of the magnetic grains, Drs. J. Souletie and K. Matho for general discussions, as well as Drs. S. Hunklinger and M. Von Schikfuss from the Max-Planck-Institut, who also provided the sample. We also acknowledge J. C. Le Gilchrist for useful discussions and for checking the absence of field dependence at $4.2 \mathrm{~K}$.

\section{References}

[1] Stephens, R. B., Phys. Rev. B 8 (1973) 2896;

lasjaunias, J. C., Ravex, A., Vandorpe, M. and HunkLINGER, S., Solid State Commun. 17 (1975) 1045.

[2] Piche, L., Maynard, R., Hunklinger, S. and Jackle, J., Phys. Rev. Lett. 32 (1974) 1426.

[3] Von Schickfuss, M., Hunklinger, S. and Piche, L., Phys. Rev. Lett. 35 (1975) 876.

[4] Anderson, P. W., Halperin, B. I. and Varma, C. M., Phil. Mag. 25 (1972) 1;

Phillips, W. A., J. Low Temp. Phys. 7 (1972) 351.
[5] Bakalyar, D., Swinehart, R., Weyhmann, W. and LawLESs, W. N., Proc. LT 13, Boulder, p. 646, vol. 4, J. D. Timmerhaus ed. (Plenum Press); Rev. Sci. Instrum. 43 (1972) 1221.

[6] JACKLE, J., Z. Phys. 257 (1972) 212.

[7] PIRC, R. and Gosar, P., Phys. Kondens. Mater. 11 (1970) 163; SANDer, L. M. and Shore, H. B., Phys. Rev. B 4 (1971) 1472.

[8] Néel, L., J. Physique Radium 11 (1950) 49.

[9] Maynard, R. and Rammal, R., to be published.

\section{REMARQUE DE L'ÉDITEUR}

Nous tenons à préciser que la note figurant à la fin de l'article intitulé :

« Les microémulsions relèvent-elles des phénomènes polycritiques? »

paru dans le Journal de Physique Lettres, tome 38 (1977), $1^{\text {er }}$ mars, page L-137 est une note de l'Editeur. 